\title{
LA EDUCACIÓN JURÍDICA COLOMBIANA Y LA GLOBALIZACIÓN: ENTRE LOS ESTUDIOS DE "CAJA NEGRA”, EL FORMALISMO JURÍDICO Y LA NUEVA HEGEMONÍA
}

\author{
COLOMBIAN LEGAL EDUCATION AND \\ GLOBALIZATION: AMONG THE STUDIES \\ OF “BLACK BOX”, LEGAL FORMALISM \\ AND THE NEW HEGEMONY
}

Juan Pablo Sarmiento E*

\begin{abstract}
PARA CITAR ESTE ARTículo / To CITE THIS ARTICLE Sarmiento, J., La educación jurídica colombiana y la globalización: entre los estudios de "caja negra", el formalismo jurídico y la nueva hegemonía, 24 International Law, Revista Colombiana de Derecho Internacional, 59-82 (2014).

doi:10.11144/Javeriana.IL14-24.ejcg
\end{abstract}

* Abogado de la Pontificia Universidad Javeriana, Magíster y Doctor en Derecho de la Universidad de los Andes, Bogotá, Colombia. Profesor e investigador de la Universidad del Norte, Barranquilla, Colombia. Este trabajo es resultado de una investigación financiada por la Universidad del Norte, sobre globalización y derecho, en el Grupo de Investigación de esta universidad en Derecho. Correos electrónicos: jpsarmiento@uninorte.edu.co,jua-sarm@ uniandes.edu.co 


\section{RESUMEN}

El presente documento realiza una aproximación a la forma como parecen "reaccionar" las principales facultades de derecho en Colombia, en función de lo que hemos entendido por "globalización". Para este efecto, se abordará una visión crítica de las convenciones alrededor de la globalización, desde la teoría general o transnacional del derecho, para luego ofrecer un panorama de los planes de estudio de las universidades que, según hemos considerado, pueden ser representativas de la experiencia nacional. La metodología que hemos utilizado consiste en un análisis comparativo de los planes de estudio de las universidades seleccionadas, se ha examinado cuáles facultades ofrecen asignaturas que pueden contener una visión globalizada del derecho, y cuáles presentan una dominancia en las asignaturas "profesionalizantes" o de "caja negra". La conclusión que se presenta da cuenta de la preponderancia que tienen las materias locales y que se encuentran enquistadas en la teoría particular del derecho.

Palabras clave autor: Educación jurídica, globalización, derecho, facultades de derecho y globalización.

Palabras clave descriptores: Derecho, enseñanza, globalización. 


\begin{abstract}
This paper is an approach to the way major law schools in Colombia seem to "react", according to what we understand by "globalization". To this effect, a critical view of the conventions around globalization will be addressed, from general or transnational legal theory, to then provide an overview of the curricula of the universities, as we have seen, may be representative of the national experience. The methodology we have used is a comparative analysis of the curricula of selected universities, we have examined what schools offer courses that can contain a global view of the law, and which have dominance in the "vocational" or "black box" subjects. The conclusion presented accounts for the preponderance with local subjects and are entrenched in the particular legal theory.
\end{abstract}

Keywords author: Legal education, globalization, law, law schools and globalization.

Keywords plus: Law, study and teaching, globalization.

SUMARIO

INTRODUCCIÓN.- I. LA GLOBALIZACIÓN Y EL DERECHO: REPLANTEAR LA TEORÍA TRADICIONAL DEL DERECHO.- II. EL ETERNO RETORNO AL FORMALISMO COMO FUENTE DE PRODUCCIÓN Y ENSEÑANZA DEL DERECHO.- III. LAS FACULTADES DE DERECHO, ENTRE LA DICOTOMÍA DEL DERECHO INTERNACIONAL, LA “CAJA NEGRA" Y EL AislaCionismo.- IV. CONCluSión.- Bibliografía 
INTRODUCCIÓN

La globalización, como se procederá a exponer, no es un fenómeno novedoso. Su significación ha variado de manera continua, carece de un concepto integrador y discernible y solo recientemente algunas facultades de derecho nacionales parecen haberle prestado atención. En efecto, el presente artículo pretende realizar una aproximación a la forma como las facultades de derecho en Colombia podrían estar "reaccionando" a la transformación que supone la globalización para el Derecho.

Por supuesto, hacer uso del término "globalización" en un estudio sobre derecho puede ser problemático, pues se trata de un fenómeno multifactorial ${ }^{1}$ que puede ser visto desde distintos ángulos, disciplinas y paradigmas, y que no pertenece a ninguna categoría jurídica. Autores como Duncan Kennedy presentan una visión panorámica de lo que puede ser la globalización, y argumentan que en realidad han existido "olas" de globalización que trascienden las instituciones jurídicas, aunque en buena parte determinan su contenido desde estrategias graduales y van acompañadas de vicisitudes económicas, políticas, sociales e incluso ideológicas ${ }^{2}$.

Esta visión crítica sugiere que la globalización instrumentaliza las normas jurídicas y las convierte en herramientas que adjudican y moldean las instituciones transnacionales, por tanto, esto conduce a que el derecho interno sea solo un reflejo de agendas políticas transfronterizas. Sin embargo, la tercera ola de globalización que, según los cálculos, es la que actualmente se ha desplegado, se muestra mucho más agresiva en cuanto a la subjetividad política, tanto individual como nacional, pues no solo trasciende

1 I. Ordorika Sacristán, Educación superior y globalización: las universidades públicas frente a una nueva hegemonía, 3 Revista Andamios, 5, 31-47, (2006). El autor identifica al menos tres esferas significativas: economía, sociedad y cultura, empero, puede resultar "econocentrista" por cuanto considera que las principales manifestaciones de la globalización se encuentran en el dominio del sistema de producción capitalista que se nutre del uso de tecnologías de la información y la comunicación.

2 Ver D. Kennedy, Three Globalizations of Law and Legal Thought: 1850-2000, en The New Law and Economic Development. A Critical Appraisal, 19-73 (Cambridge University Press, Boston, 2006). 
fronteras: también es transcultural, y tiende a permear de forma significativa el derecho interno, lo que prácticamente convierte en obsoletos los discursos sobre la soberanía, la identidad nacional y el monopolio en la producción del derecho, que antes ostentaba el Estado-nación ${ }^{3}$.

La aproximación más cercana a esta tercera ola de la globalización fue captada en principio por la construcción dicotómica del derecho internacional público y el derecho interno ${ }^{4}$. Esta visión entendería que la soberanía se podría reducir o relativizar por medio de tratados internacionales que, bajo un paradigma privatista, fueran capaces de negociar derechos de los Estadosnación y ceder, solo hasta cierto punto, la producción del derecho a organismos y tratados transnacionales.

Sin embargo, autores como William Twining y, desde la experiencia nacional, Oscar Guardiola, Clara Sandoval y Diego López, encuentran que esta dicotomía resultaría precaria, pues se trata de una proposición limitada, en tanto la globalización de finales del siglo XX e inicios del siglo XXI supera la visión de la posguerra fría del "nuevo orden mundial". Los autores citados sostienen que en la experiencia contemporánea la globalización actuaría de formas más soterradas, con la capacidad de articular dinámicas neocolonizadoras que extinguen las subjetividades políticas y extienden una cultura hegemónica por fuera de las instituciones jurídicas.

Por supuesto, el estudio que ahora se expone no pretende juzgar las ventajas o desventajas de este proceso, ni cuestionar en lo moral o ético sus consecuencias. Aquí mostraremos, en primer lugar, la globalización, sus demandas y consecuencias para el derecho; y a continuación, daremos una mirada a las visiones críticas dadas al formalismo, como fuente de convenciones del lenguaje jurídico e insumo de los discursos obsoletos, así como el estudio de "caja

3 Ver O. Guardiola Rivera \& C. Sandoval Villalba, Un caballero inglés en la corte del Gran Khan. En torno a los estudios sobre globalización y derecho de William Twining en Derecho y Globalización, 68 y ss. (Uniandes, Siglo del Hombre, Instituto Pensar, Bogotá D. C., 2003). Ver también J. De Lucas, Globalización e identidades. Claves políticas y jurídicas, 70 y ss (Icaria Editorial S. A., Barcelona, 2003).

4 O. Guardiola Rivera \& C. Sandoval Villalba Op. cit., p. 80. 
negra". A partir de lo anterior será posible examinar el currículo de las principales facultades de derecho del país, explorar las limitaciones, aproximaciones o consecuencias que podrían tener para el jurista en formación, así como la visión dominante del derecho que se transmite en la enseñanza jurídica contemporánea.

\section{LA GLOBALIZACIÓN Y EL DERECHO: REPLANTEAR}

LA TEORÍA TRADICIONAL DEL DERECHO

En este título se abordarán dos subtesis centrales para realizar la aproximación a la educación jurídica como objeto de estudio. La primera se referirá a las consecuencias de la globalización desde autores como Diego López, Oscar Guardiola, Clara Sandoval, William Twining y Gunther Teubner; en segundo lugar, la demanda por una teoría transnacional del derecho, capaz de contener una teoría unificada que, sin importar la "cartografía"s de los ordenamientos jurídicos, permite explicar lo que el derecho es y hace.

En la actualidad, podríamos afirmar, existe una multiplicación exponencial de fuentes del derecho que, es evidente, han disuelto buena parte del ordenamiento jurídico interno, han impuesto agendas políticas transnacionales y han removido instituciones públicas que pudiesen considerarse "particulares" para el orden jurídico interno. Empero, la visión positivista del derecho - que aún domina en la educación jurídica colombiana y que permea los planes de estudio de las facultades de derecho seleccionadas - dejaría de lado un atributo que parecería ineludible para el jurista en el contexto de la globalización: el derecho global tiene la capacidad de trascender culturas, y fronteras ideológicas e institucionales.

5 W. Twining, Cartografiar el derecho, en Derecho y globalización, 173-207 (Siglo del Hombre, Uniandes, Instituto Pensar, Bogotá D.C., 2003). Con el término "cartografía" hago alusión a la posición crítica adoptada por Twining, según el cual existen a escala global, cuando menos, diferentes "familias legales", a saber: la romana, la germánica, la nórdica, la de derecho común, la socialista, los sistemas del Oriente lejano, los sistemas islámicos y el derecho indio. Con todo, el autor reconoce que esta labor, además de difícil, resulta problemática y un poco estéril, pues demanda que el investigador pueda trazar fronteras y definir sistemas "puros", pero que al final, dan cuenta de "trasplantes", mezclas y carencia de criterios, incluso, para definir si ciertas prácticas sociales son o no derecho. 
Para probar este punto, basta señalar que la teoría general del derecho que todavía se enseña en las facultades, suele exponer las principales escuelas de pensamiento jurídico — del norte global o de experiencias dominantes como la europea y estadouniden$\mathrm{se}^{6}$-, entre el ius naturalismo, el positivismo, el formalismo, el neoconstitucionalismo y, en algunos, los estudios críticos del derecho que, por demás, hacen parte de un discurso hegemónico de la globalización. Estos constituyen al derecho como articulador y regulador de la vida social, "natural", que se mantiene como la única posible, homogénea y aceptada por todos?. Por supuesto, se trata de una nominación general de las principales escuelas del pensamiento jurídico, que permite explicar el derecho continental u occidental, y el derecho nacional o particular, y que incluso logra demostrar la existencia de teorías transnacionales, bajo el amparo de autores prestigiosos, emblemáticos en las escuelas de derecho colombianas, entre otros, Hans Kelsen, H. L. A. Hart, Ronald Dworkin y Robert Alexy. Es claro que la relevancia del estudio de la teoría general del derecho no se descarta, sin embargo, demuestra a simple vista una limitación significativa, a saber: ninguna de estas corrientes de pensamiento permitiría razonar el derecho oriental, islámico o socialista y, algunas veces, podría ser insuficiente para comprender el derecho anglosajón y la "familia de derecho común", aunque occidental.

Los autores "prestigiosos" citados y el contenido de sus construcciones dogmáticas no son objeto de estudio del presente

6 Esto sin descontar que esas corrientes de pensamiento emergieron de "prestigiosas" escuelas de derecho, que por diversas razones políticas, sociales, económicas y culturales se convirtieron en estandartes del pensamiento jurídico. Según Ordorika, la educación superior es un campo disparejo, jerárquico, cambiante y en disputa permanente, que se ha acercado cada vez más a la lógica de mercado. Expone: "El mercado está estructurado en dos niveles. Por un lado, una 'super-liga' de universidades globales de investigación, que persiguen fundamentalmente prestigio y poder, más que ganancias económicas como tales. Por otro lado, hay un grupo más amplio de instituciones con menos estatus, involucradas en la exportación comercial de la educación superior, con un modelo de desarrollo de expansión capitalista. Este mercado global está mediado por tablas comparativas de desempeño en investigación o estatus universitario. Los 'ranqueos' internacionales más conocidos son los producidos por la Shanghai Jiao Tong University y el UK Times Higher Education Supplement". I. Ordorika Sacristan, Óp. cit., pp. 31-47. La autoría citada referencia a S. Marginson \& M. Considine, The Enterprise University: Power, Governance, and Reinvention in Australia (Cambridge, University Press, 2000).

7 W. Twining, Óp. cit., pp. 173-207. 
documento, pero se agrupan en torno al derecho occidental continental. Esto implica, por supuesto, una ideología que permea inclusive el estudio y la producción de documentos jurídicos, y supone que la globalización, al extraer el monopolio de la producción de derecho por el Estado, consigue que estas escuelas del pensamiento jurídico pierdan uno de sus insumos, por lo menos, el positivismo, el formalismo jurídico y el neoconstitucionalismo, y con ello, la capacidad analítica, por parte del jurista en formación, en términos del conocimiento y competencias transmitidas.

La multiplicidad de fuentes del derecho, la disolución de las fronteras políticas y el multiculturalismo demuestran la deficiencia y las limitaciones de las herramientas analíticas que ofrecía la tradicional teoría general del derecho y la escuela de pensamiento jurídico dominante en nuestra práctica, así como las materias que, por ahora, llamaremos "profesionalizantes"; además, hace evidente la ruptura con las fuentes tradicionales de conocimiento jurídico.

Más allá de la demanda por una conceptualización o teorización del derecho, las transformaciones contemporáneas y el derecho global nos conducen a repensar la educación jurídica. Sin duda, la invitación al debate sobre los retos de la globalización y el derecho supone, por una parte, la construcción de herramientas analíticas que soporten estudios del derecho como ciencia; por otra parte, implica la revisión del contenido mismo de las asignaturas, que parecerían haber sido diseñadas por la visión formalista como teoría dominante del derecho colombiano. Estas han eludido la teoría del derecho en el contexto de la globalización, en tanto esta última pudiese ser una herramienta cuya carga política, sociológica

8 El término "profesionalizante" se refiere a la acostumbrada y dominante producción y enseñanza del derecho, que ha sido llamado el estudio normativista o aislacionista del derecho. Propone la descripción del ordenamiento jurídico y sus vicisitudes como un desarrollo natural de unas normas hacia otras; aboga por la autonomía absoluta del derecho, pues entiende que esta disciplina es fuente de conocimiento independiente y autorreferente, deja de lado el entorno, los debates oficiales y las funciones social y política insertadas primigeniamente en la institución jurídica objeto de análisis. Sin embargo, esta manera de explorar y exponer la institución analizada trae consigo un problema de limitación sobre el objeto de estudio y esconde la carga ideológica en la producción del derecho. Ver J. P. Sarmiento, La justicia administrativa entre dictaduras, una crítica a la construcción del derecho administrativo en Colombia (Tesis sin publicar, para optar al título de Doctor en Derecho, Universidad de los Andes, Bogotá D.C., 2012). 
e ideológica no parecería admitir. La globalización exigiría al derecho, a su teoría general y a su enseñanza, un redireccionamiento capaz de afrontar la pluralidad de fuentes jurídicas, las teorías transnacionales del derecho y la reconquista de espacios analíticos monopolizados por otras ciencias sociales, como la ciencia política, antropología, sociología, historia, sociología, etc.

Lo anterior, con base en que la globalización, si bien se trata de un fenómeno con larga historia, ha experimentado una intensificación reciente de las relaciones jurídicas y políticas, situadas en los extremos más poderosos o dominantes y los más pobres o subalternos, que hoy han dejado de ser mediadas por los Estados-nación; de este modo, se ha diluido la subjetividad política, y con ello la individualización de los ordenamientos jurídicos de las naciones. La diversidad de relaciones jurídicas entre los sujetos de derecho parece vaciar la soberanía del Estado en la aparente soberanía individual, y replantear los pilares del derecho estatal que estudiamos desde el formalismo jurídico imperante en las facultades colombianas de derecho.

La transformación institucional subyacente a la globalización parece ser una tarea que las teorías tradicionales y la enseñanza formalista del Derecho no pueden asumir. Inclusive, de acuerdo con la expresión de Twining, es imperioso abandonar la "caja negra", esto es, las parcelaciones de las experiencias comparadas para dar paso a las teorías e instituciones jurídicas globales, sin dejar de lado, por supuesto, las consecuencias y relaciones de poder implícitas 9 , ni las adaptaciones y orientaciones que las prácticas nacionales pueden haber realizado.

Esto, sin olvidar que el estudio del derecho en el contexto de la globalización no depende de la dicotomía entre el derecho estatal y el derecho internacional público o las instituciones jurídicas transnacionales con sus vicisitudes y alcances, como parecen haberlo interpretado algunas facultades que más adelante resaltaremos. La globalización parece demandar la reestructuración de las fuentes del derecho, la explicación de instituciones jurídicas que

9 W. Twining, Ibídem, pp. 122 y ss. 
se desplazan y atraviesan jurisdicciones, fronteras, tradiciones y culturas para ampliar el objeto de estudio, empobrecido por la enseñanza del derecho desde teorías jurídicas particulares y asignaturas que hemos llamado, "profesionalizantes". Opina Teubner que la globalización es un proceso policéntrico - en que diversos ámbitos vitales superan sus límites regionales y constituyen respectivamente sectores globales autónomos-, entendido como un "fenómeno multidimensional que implica diversas áreas de actividad e interacción, incluyendo el campo económico, político, tecnológico, militar, cultural y medioambiental" 10 .

Esta propiedad y consecuencia de la globalización provoca una reconceptualización más significativa aun, pues se trata de un severo límite trasmitido desde la enseñanza a la práctica de los estudiantes de derecho. Aunque se trate de un sistema educativo que aboga por otro tipo de docencia, más allá del formalista o vertical, y se ofrezcan competencias cognoscitivas y prácticas, el límite se transmitirá a partir del objeto de estudio de las materias que consideramos centrales, sus contenidos y la producción bibliográfica que se ha producido en torno a ella.

Lo anterior conduce a replantear la aproximación a las asignaturas en derecho, como fundamentación del campo jurídico del estudiante en formación, para abordar las instituciones locales a partir de la teoría comparada del derecho, que consigue hallar principios, nociones y distinciones comunes de las experiencias más "maduras"11. Con todo, la reconstrucción teórica y práctica que las facultades de derecho nacionales parecen desplegar, estarían siguiendo otro rumbo en tanto se confinan en un sistema jurídico nacional o particular, desde una visión formalista, muy a pesar de la demanda de juristas capaces de sobrellevar la enorme carga de la multiplicación de fuentes jurídicas.

De allí que la alternativa planteada por Oscar Guardiola, Diego López y William Twining proponga el tránsito de la teoría

10 G. Teubner, Regímenes globales privados: ¿derecho neoespontáneo y constitución dual de sectores autónomos? en Estado, soberanía y globalización, 67 y ss. (Siglo del Hombre, Uniandes, Pontificia Universidad Javeriana-Instituto Pensar, Bogotá D. C., 2010).

11 D. López Medina, Teoría impura del derecho: la transformación de la cultura jurídica latinoamericana, 375 y ss. (Legis, Bogotá D. C., 2004). 
general del derecho a la teoría comparada, esta última, soporte de la teoría transnacional del derecho. Ahora bien, la crítica planteada por los autores citados reconoce, de entrada, que las teorías transnacionales y transculturales del derecho carecen de capacidad explicativa, en la medida en que sus conceptos, categorías y fronteras determinan sus límites. Ciertamente, las convenciones del lenguaje jurídico imponen restricciones que impiden la formulación de esta teoría. Sin embargo, parece necesario incluir, en cuanto al estudio del derecho, una teoría capaz de ofrecer un marco analítico que permita encontrar los fines y temas comunes a los ordenamientos jurídicos, y quizá, a las "familias legales", como puntos convergentes; por supuesto, este se referirá a unas cuantas experiencias comparadas y diferenciará los centros de producción del derecho y las experiencias receptoras. Así, la propuesta o crítica que ahora se recoge no se centra en una reforma o inflación de la teoría general o comparada del derecho, pues, según las críticas de Diego López a la propuesta de Austin, no podemos presuponer la existencia de una naturaleza general del derecho, vinculada de modo analítico a la naturaleza del ser humano ${ }^{12}$.

Por supuesto, los argumentos que hasta ahora se han recogido suponen una severa crítica a los límites contextuales y geojurídicos, que se transmiten ante la masiva y dominante producción de textos jurídicos, evidente en ciertas áreas del derecho, donde las discusiones sobre la teoría general se diluyen en su enseñanza "profesionalizante" y su teoría particular, la caja negra, en términos de Twining.

La teoría transnacional del derecho da cuenta de los trasplantes jurídicos, los contextos de producción y los sitios de recepción, pero niega la necesidad de explicar la cultura jurídica propia, sus prácticas y posibilidades. Inclusive, afirma el profesor López en su lectura crítica de la teoría transnacional del derecho, puede llegar a ser permeable a cierta dinámica neocolonizadora relativa a la filiación con determinado ordenamiento jurídico, y a la incapacidad de formular una teoría general del derecho transcultural.

12 D. López Medina, Óp. cit. 
Como bien lo ha señalado López en varias de sus obras, la teoría transnacional del derecho parece un artefacto ligado a una cultura neocolonial, más que una herramienta vinculada con exigencias epistemológicas del pensamiento jurídico. A fin de cuentas, el debate se despliega en torno a un contexto distinto al que permitió la emergencia de las teorías del derecho a mediados del siglo XX. La globalización impone un deber de reformulación ius teórica y un reto a la comprensión, por tanto, a la enseñanza del derecho ${ }^{13}$.

De allí que la globalización y los estudios jurídicos transnacionales replanteen una discusión fundamental que se ha diluido en el formalismo jurídico, aún imperante en nuestra práctica $\mathrm{y}$, sobre todo, en nuestra enseñanza y producción de textos jurídicos. La cuestión sobre la cientificidad y la metodología para la producción y transmisión del conocimiento en derecho parece esquiva a los aportes más recientes de la teoría general del derecho, que se enseña en nuestras facultades.

\section{EL ETERNO RETORNO AL FORMALISMO COMO FUENTE} DE PRODUCCIÓN Y ENSEÑANZA DEL DERECHO

Los debates en torno a la producción y enseñanza del conocimiento jurídico parecen esquivos y se reducen en medio de textos formalistas, relativamente útiles para el estudio del derecho como práctica. En efecto, la producción dominante de documentos jurídicos habría vaciado su método de estudio y comparte ahora fuentes de producción del "conocimiento jurídico" con la ciencia política, la antropología, la historia y la economía, entre otras ciencias sociales. El derecho carece ahora de un método y los textos jurídicos se desarrollan a partir del derecho como técnica y no como ciencia social. Este aislamiento trae como consecuencia un empobrecimiento significativo del estudio del derecho y de la manera como este se imparte en las facultades de derecho.

La educación jurídica contemporánea seguiría "conquistada" por las tres falacias propias de la "insuperable" cultura formalista

13 D. López Medina, Ibídem, p. 376 
en derecho, a saber, la forma lógica y el silogismo práctico jurídico; segunda, la separación entre derecho, moral y política; y tercera, el elevado y aséptico análisis judicial ${ }^{14}$. Quizá se trate de discusiones basadas en experiencias dominantes, cuyo tránsito parecería inminente en cuanto a reconstruir la producción jurídica formalista, a partir de las cenizas que deja la globalización de las teorías jurídicas modernas (de inicios y mediados del siglo $\mathrm{XX}$ ), enquistadas en nuestra formación jurídica tradicionalista.

El empobrecimiento metodológico del derecho, termina por suponer que este ha sido incapaz de producir su propio estatuto epistemológico y que acude a otras disciplinas como fuentes epistémicas para abordar el derecho como objeto político. Son pocos los estudios y las facultades que han abordado la quizá inevitable, "pluridisciplinariedad del campo de estudio"15.

Con todo, la producción y formación jurídica parece enclaustrada en una visión que resguarda la "autonomía absoluta" del derecho y su producción aislacionista, resultado de la llamada "desviación técnica". Explica Michel Miaille que esta "desviación" es una expresión clara del triunfo formalista que convierte el estudio de las "técnicas jurídicas" en la esencia del razonamiento del derecho. Esta posición epistemológica permite evacuar, casi por completo, todo aquello que no sea jurídico; de esta manera, el texto o documento en derecho cumple su tarea de reportar exclusivamente el contenido normativo del sistema jurídico, no así aquel documento que plantee problemas sociológicos, históricos o económicos. Se trata entonces de una "ideología tecnocrática", que se presenta como neutral o "no ideológica", en tanto los juristas se comprometen con la aplicación de un derecho que excluye el razonamiento sobre la expresión del poder político que contiene. La mitificación de un derecho autónomo, técnico y neutral permite hacer a un costado los partidos, los grupos de presión y, en general, el entorno histórico, económico y político para, finalmente, atribuirle al derecho una propiedad que no tiene ${ }^{16}$.

14 Ibid., pp. 11-22.

15 J. P. Sarmiento, Óp. cit., p. 10.

16 Ver M. Miaille, Les figures de la modernité dans la science juridique universitaire, en Pour 
Ante los retos de la globalización, las obras y la enseñanza del derecho que tiendan a suponer que existe un derecho autorreferente se pueden considerar riesgosas, con fuentes que pueden obviar la lectura de realidades sociales y globales, y que se agota en sus propias instituciones, cuyo desarrollo puede ser comprendido solo por medio de su "dinámica interna"17. Con esta explicación no se quiere tratar de forma peyorativa a las obras o facultades que puedan encajar con esta descripción, en cuanto consideren en su conjunto que el derecho es una técnica, y como tal su estudio se agota con la calificación e interpretación de categorías jurídicas, el precedente judicial, normas y principios vigentes, de utilidad relativa para resolver litigios o cuestiones sobre el alcance normativo del texto jurídico.

No obstante, la amplia extensión de producciones bibliográficas y asignaturas que se destinan a reproducir el estudio del derecho desde su autonomía absoluta y, por tanto, que contienen lecturas empobrecidas de las instituciones jurídicas, tiende a arrojar consecuencias diversas a la aplicación y creación misma del derecho. Ciertamente, el aislacionismo y el estudio de "caja negra" conduce a las tres consecuencias nocivas, dos enunciadas por el autor Diego López Medina: consigue que el jurista aplique y agregue a su conocimiento instituciones desprevenidamente de su origen o de los procesos políticos que les subyacen, y por consiguiente, los observe como neutrales; segundo, niega el clima clientelista y la participación de actores políticos y agentes económicos, en consecuencia, niega reflexiones políticas,

une critique du droit, 141-142 (François Maspero, Coord., Presses Universitaires de Grenoble, 1978). Ver también G. Teubner, Elementos materiales y reflexivos en el derecho moderno, en La fuerza del derecho, 142 (Nuevo Pensamiento Jurídico, Siglo del Hombre, Pontificia Universidad Javeriana, Uniandes, Bogotá D.C., 2005). En el segundo texto citado, Teubner se hace una profunda crítica sobre el formalismo jurídico. Sostiene el autor: "[l]a racionalidad legal formal y la jurisprudencia sociológica eran fundamentalmente incompatibles porque la primera consideraba los actores legales como desligados del mundo, permitiendo de esta forma la afirmación de que tomar en cuenta los aspectos sociales, económicos y políticos no era una tarea \para un abogado como tal" (Resaltado en el original). Para sustentar esta afirmación, el autor cita a Windscheid Bernhard, Die Aufgaben der Rechtswissenschaft, 101 (Leipzing Duncker, 1904).

17 Ver P. Bourdieu, Raison pratiques. Sur la théorie de l'action, 156 (Éditions du Seuil, París, 1994). 
económicas y sociales ${ }^{18}$. Un tercer efecto nocivo es que tiende a desagregar los discursos y las aproximaciones de las agendas transnacionales y la recepción, no solo de teorías del derecho sino de instituciones jurídicas, que en ocasiones se consideran útiles, debido a que provienen de naciones "más civilizadas", por lo general, del norte global.

Esto último lleva a que el jurista moderno esté "cegado" con las formas $\mathrm{y}$, por tanto, pierda toda perspectiva de temporalidad o profundidad del derecho como discurso, y niegue, en particular, la propiedad ideológica de esta ciencia social aplicada. Tal situación es de suma gravedad para cualquier país en el contexto de la globalización, pero más preocupante para culturas como las iberoamericanas latinoamericanas o en desarrollo, en cuanto extravía la dimensión social, el conocimiento del derecho desde sus orígenes y las agendas transnacionales, la mayoría de las veces, neocolonizadoras. La sustracción del derecho a una clase de personas competentes, los juristas, que conocen de modo exclusivo el derecho local y fueron formados en "cajas negras", y quienes se hacen los intérpretes autorizados del contenido normativo, supone también la pérdida del carácter óntico del derecho como un instrumento propio de la naturaleza de la sociedad, que se descubre y se lee en la realidad social y se traduce en reglas.

La propiedad del derecho que lo hace permeable a decisiones políticas transfronterizas y locales, versa sobre su aplicación en campo, escenario donde la forma jurídica puede abrir, contener o cerrar su propia materia. El derecho no puede ser reducido a una materia neutra ni ostentar esa propiedad, puesto que guarda un lenguaje mucho más alterable por la política, y esto permite que esta última actúe de forma cómoda con la sensibilidad social, la hegemonía global y la indeterminación de las categorías jurídicas, lo que hace de la tarea hermenéutica jurídica, una

18 D. López Medina, El sueño weberiano. Claves para una comprensión constitucional de la estructura administrativa del Estado colombiano, 1 Revista de Derecho Público, 1, 2-36 (2006). 
herramienta necesaria para introducir orientaciones y valores políticos en los textos jurídicos.

La anterior descripción nos induce al cuestionamiento epistemológico más crítico para el contexto actual de producción y enseñanza del derecho, que reconocemos como globalización. En la medida en que los conceptos tradicionales de Estado, nación, ciudadanía, Constitución y derecho han sido puestos a prueba por la globalización, que la soberanía de los Estados se ha disgregado en medio de la complejidad de relaciones supranacionales y que las fuentes del derecho se han multiplicado, el estudio de este demanda no solo una reconceptualización como disciplina: supone un reto significativo para comprenderlo como una ciencia que exige análisis comparados, contextualizados y multifactoriales.

El estudio tradicional del derecho ha sido desafiado, ha perdido su capacidad explicativa, se ha disuelto en reinterpretaciones locales de fenómenos globales y viceversa. La experiencia latinoamericana y, en especial, la colombiana, no ha sido la excepción. La multiplicidad de fuentes del derecho, la disolución de las fronteras políticas, el multiculturalismo y las teorías transnacionales del derecho han permeado nuestro ordenamiento jurídico y lo han direccionado hacia la ruptura con las fuentes tradicionales de conocimiento jurídico. Así, nos encontramos ante un objeto y un período de estudio privilegiado, permeable a decisiones multilaterales pero con un contexto excepcional, que ubica a Colombia como un productor único de instituciones jurídicas tan particulares como su realidad, pero a la vez, como un Estado que funde sus fronteras en el derecho global.

En efecto, la suscripción de tratados de libre comercio, la inclusión en mercados transnacionales y los documentos internacionales sobre derechos humanos, derecho internacional humanitario y derechos económicos, sociales y culturales, han sumergido al país en una inmensidad de productores de derecho, que parecen quedar relegados desde la educación y producción jurídica dominante, e ignorar la manera en que ciertas prácticas regulatorias nacionales han creado las condiciones necesarias 
para la globalización y el aseguramiento de los mercados transnacionales de capitales ${ }^{19}$.

\section{LAS FACULTADES DE DERECHO, ENTRE LA DICOTOMÍA DEL DERECHO INTERNACIONAL, LA "CAJA NEGRA" Y EL AISLACIONISMO}

En Colombia existen alrededor de 75 facultades de Derecho, aunque solo 26 están acreditadas por el Consejo Nacional de Acreditación-Ministerio de Educación Nacional. Por supuesto, se trata de una cifra que quizás no sea tan precisa, pues solo cuenta facultades calificadas por el Consejo. No obstante, para el presente estudio y por razones de tiempo y espacio, se ha hecho una selección basada en los siguientes criterios: prestigio, número de estudiantes y posición geográfica. En este sentido, este documento incluye las siguientes universidades: en Bogotá, Pontificia Universidad Javeriana, Universidad de la Sabana, Universidad Nuestra Señora del Rosario, Universidad Externado, Universidad de los Andes, Universidad Nacional, Universidad Libre; en Medellín, Universidad de Medellín, Universidad EAFIT, Universidad de Antioquia; en Cali, Universidad ICESI; en Barranquilla, Universidad del Norte; y en Cartagena, Universidad de Cartagena.

Se espera encontrar entre estas las similitudes y diferencias en los programas de derecho, para así identificar el dominio de materias que puedan dar cuenta del estado de la educación jurídica a escala nacional ante la globalización. De igual manera, se busca cubrir las principales ciudades del país, por su población, número de estudiantes activos y relevancia económica, esto es, se conocerán los programas de Derecho en Bogotá, Medellín, Cali, Barranquilla y Cartagena.

Adicional a lo antedicho, se parte de la premisa de que las facultades seleccionadas se han preocupado por actualizar sus

19 S. Sassen, Desnacionalización de las políticas estatales y privatización de la producción de normas, 103-122 (Siglo del Hombre, Bogotá D. C., 2010). 
programas bajo la idea de la "internacionalización del derecho", y quizá con ello, la globalización. En cuanto al prestigio académico, todos estos centros de estudio están certificados ante el Consejo Nacional de Acreditación, inclusive, tienen programas de intercambio estudiantil en pregrado con universidades estadounidenses, europeas y latinoamericanas. Sin embargo, en la medida en que son opcionales, su oferta está condicionada a requisitos académicos y económicos, que pueden resultar ajenos al objeto de estudio, pues se pretende dar una vista panorámica al currículo, pero no a los programas adicionales que puedan ofrecer las facultades de Derecho.

Con esto no queremos insinuar que las facultades no incluidas aquí carezcan de prestigio $u$ ofrezcan programas obsoletos o poco rigurosos para este documento. Al contrario, como se mencionó, se tomó la muestra de algunas facultades por razones de tiempo y de espacio. Para abarcar todos los programas, a continuación se presentará un resumen gráfico con las principales materias, comunes en todas las facultades estudiadas, pues hacen parte de un "núcleo" de formación "básica" del abogado, impuesto en parte por el Ministerio de Educación Nacional, veamos:

Las facultades de Derecho de la Pontificia Universidad Javeriana, Universidad del Norte, Universidad Externado, Universidad EAFIT, Universidad de la Sabana ${ }^{20}$, Universidad ICESI y Universidad Libre hacen énfasis en Economía (incluyen materias como Microeconomía, Macroeconomía, Economía Colombiana, Derecho Económico, Contabilidad, Hacienda Pública, entre otras) que no supone un título adicional o un background del jurista: las facultades que las ofrecen no otorgan un título distinto al de abogado, aunque pueden ser materias que se homologan si el estudiante opta por seguir un pregrado simultáneo al de derecho. En la Pontificia Universidad Javeriana, Universidad de los Andes, Universidad Libre, Universidad de Medellín, Universidad de la Sabana y Universidad Externado aún ofrecen Derecho

20 En la Universidad de la Sabana, la materia se llama Sistemas Jurídicos Comparados y fue incluida en la reforma curricular de 2013 para estudiantes de noveno semestre. Esto quiere decir que esta materia aún no se imparte de forma obligatoria. 


\section{Romano en sus cátedras iniciales, como medio de introducción al Derecho Civil Continental (derecho civil personas, contratos, bienes y sucesiones).}

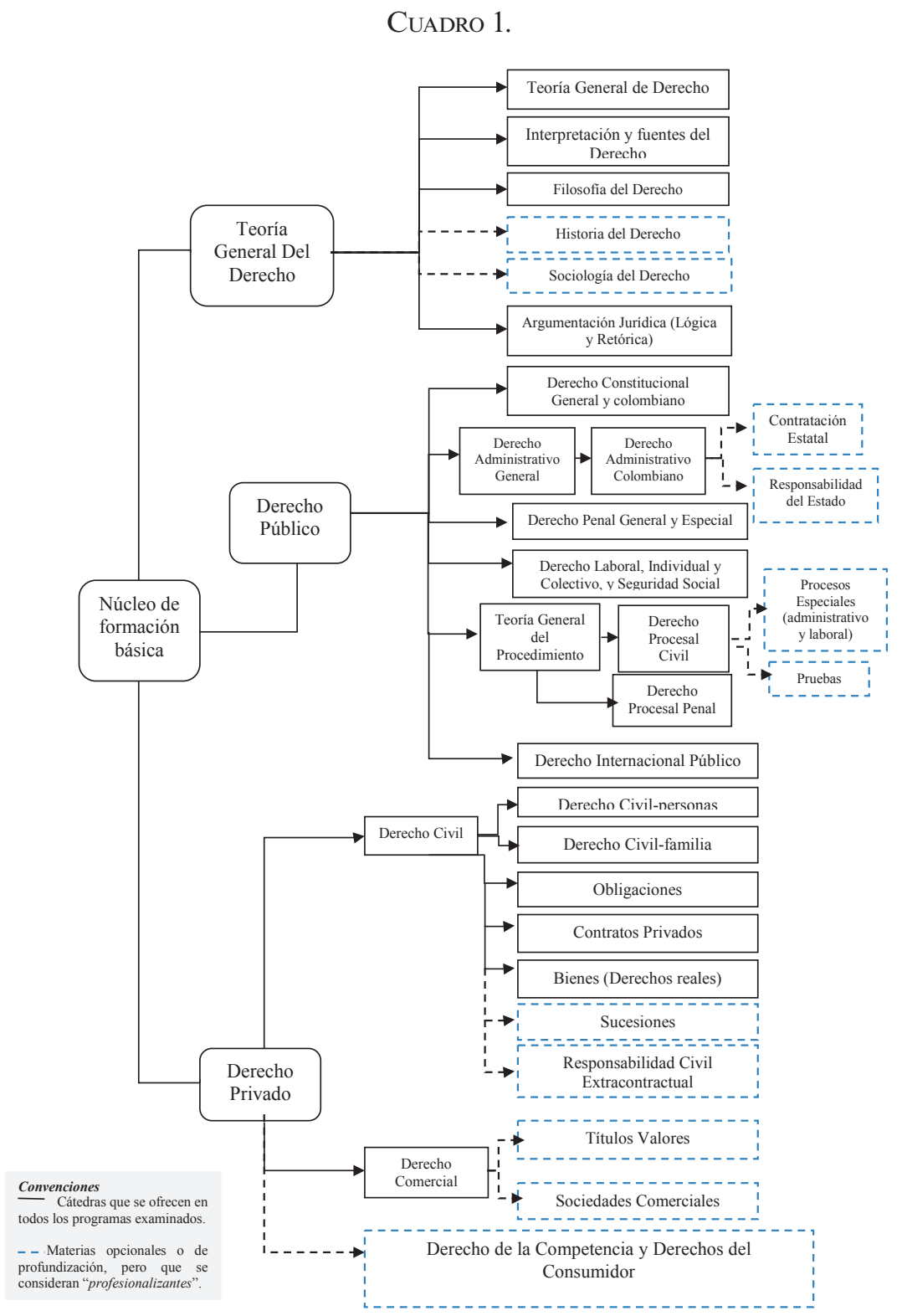

Elaboración propia 
En la Universidad del Rosario, Universidad Nacional, Universidad de los Andes, Universidad del Norte, Pontificia Universidad Javeriana, Universidad Libre, Universidad Externado y Universidad de Antioquia establecen como obligatoria la materia de Derecho Internacional Público. La Universidad Nacional, inclusive, ofrece una materia llamada Transnacionalización del Derecho, pero como materia opcional.

La Universidad del Norte y la Pontificia Universidad Javeriana ofrecen materias obligatorias como Derecho Ambiental. De igual forma, la Universidad de Cartagena al igual que la Universidad del Norte, ofrecen las cátedras de Derecho Marítimo y Derecho Portuario, la primera es obligatoria y la segunda optativa, por razones naturales, pues ambas se ubican en ciudades costeras, de esto se infiere que la posición geográfica supone la demanda laboral en esta área del derecho para sus egresados. La Universidad de Cartagena ha incorporado, sin embargo, la clase de Derecho comunitario, en la cual se estudian los modelos de intervención económica, como el Plan Marshall y la reconstrucción de Europa, el Plan Schumann, los conflictos bélicos y económicos europeos, la Comunidad Europea de Defensa (CED), los distintos modelos de integración en la Comunidad Europea (Zona de Libre Comercio, Unión Aduanera, Mercado Común, Unión Monetaria).

La Universidad del Rosario, Universidad de los Andes y Universidad de la Sabana han incluido la asignatura de Derecho Comparado. La Universidad del Rosario tiene en su plan de estudios una Teoría del Derecho Internacional, y junto con la Universidad del Norte y Universidad Libre, han incluido materias obligatorias, como Derechos Humanos y Derecho Internacional Humanitario.

Por su parte, la Universidad de Antioquia incluyó la cátedra opcional Investigación en Filosofía Política: Justicia y Globalización, que expone la noción de "globalización" desde el punto de vista analítico de la filosofía jurídica, el problema de la justicia en la globalización, los fundamentos filosóficos del nuevo orden global y el derecho, y los derechos y la política en tiempos de 
globalización. De igual forma, esta universidad ofrece la cátedra obligatoria de Problemas Latinoamericanos y Globales, que hace énfasis en los regímenes políticos en América Latina y la posición de América Latina en el orden mundial, esto es: seguridad, medio ambiente y desarrollo sostenible, migraciones, geopolítica latinoamericana, actores no estatales en América Latina e integración económica y de mercados en Latinoamérica. Esta es quizás una asignatura que integra el discurso de la dicotomía entre el derecho público e interno, con una aproximación neocolonialista presente en el estudio de la globalización.

Ahora bien, de acuerdo con los autores aquí citados, llegaríamos a la conclusión de que las facultades que dan prioridad a materias sobre Teoría del Derecho, es decir, Teoría General del Derecho, Interpretación y Fuentes del Derecho, Filosofía del Derecho, Historia del Derecho, Sociología del Derecho y Argumentación Jurídica, apuestan por la formación jurídica transfronteriza o transcultural del derecho. No obstante, una mirada a los programas de estas asignaturas, en especial, Teoría General del Derecho y Argumentación Jurídica, nos conducen a una conclusión diferente. En efecto, los programas de la Teoría General del Derecho común a la mayoría de facultades, giran en torno a las escuelas del pensamiento jurídico como el ius naturalismo, el positivismo jurídico, el realismo jurídico y, algunos, el enfoque marxista, las teorías críticas del derecho - estadounidense - y el neoconstitucionalismo, mientras que Argumentación Jurídica se sumerge en la forma lógica y el silogismo práctico jurídico.

La Universidad de los Andes ofrece Ética Profesional, con un enfoque situacional, acompañado del estudio detallado del Código Disciplinario del Abogado y las faltas disciplinarias allí consagradas. Por su parte, la Universidad de la Sabana establece como materia obligatoria Responsabilidad Profesional del Abogado, con una carga más jurídica que teleológica. 


\section{CONCLUSIÓN}

El panorama reconstruido hasta este momento nos permite llegar a tres conclusiones. Primero, las facultades examinadas parecen internarse en la dicotomía del derecho público internacional y el derecho interno, como respuesta a la globalización y la multiplicación de las fuentes del derecho. Segundo, la gran parte de las asignaturas se enmarcarían en lo que hemos denominado "profesionalizantes", donde se agotan los compartimentos en que se divide el ordenamiento jurídico (visto desde el objeto de regulación), que probablemente se desplieguen en el discurso aislacionista y se aproximan al estudio de las instituciones jurídicas locales. En tercer lugar, aun en las facultades analizadas, se constata que el estudio del derecho comparado y la globalización son reducidos. Ciertamente, en el currículo ocupan un espacio que se disuelve en medio de asignaturas que resultan imperativas para lo que es considerado como práctica jurídica contemporánea localizada.

El estudio sobre los programas de cada una de las materias que ofrecen las facultades podría dar como resultado parcelaciones integradoras y sugerentes, que respondan a la visión globalizada y enriquecida de cada una de las asignaturas. No obstante, el estudio que se presentó en este documento pretende constatar la forma en que, de manera general, se preparan hoy los futuros juristas colombianos, y sobre todo, las limitaciones de su formación ante los retos de la globalización. 


\section{BIBLIOGRAFÍA}

\section{Libros}

Bourdieu, P., Raison pratiques. Sur la théorie de l'action, 156 (Éditions du Seuil, París, 1994).

De Lucas, J., Globalización e identidades. Claves politicas y jurídicas, 70 y ss. (Icaria Editorial S.A., Barcelona, 2003).

López Medina, D., Teoría impura del derecho: la transformación de la cultura jurídica latinoamericana, 375 y ss. (Legis, Bogotá D. C., 2004).

Sassen, S., Desnacionalización de las políticas estatales y privatización de la producción de normas, 103-122 (Siglo del Hombre, Bogotá D. C., 2010).

\section{Contribuciones en Obras Colectivas}

Guardiola Rivera, O. \& Sandoval Villalba, C., Un caballero inglés en la corte del Gran Khan. En torno a los estudios sobre globalización y derecho de William Twining en Derecho y globalización, 68 y ss. (Uniandes, Siglo del Hombre, Instituto Pensar, Bogotá D. C., 2003).

Kennedy, D., Three Globalizations of Law and Legal Thought: 1850-2000, en The New Law and Economic Development. A Critical Appraisal, 19-73 (Cambridge University Press, Boston, 2006).

Miaille, M., Les figures de la modernité dans la science juridique universitaire, en Pour une critique du droit, 141-142 (François Maspero, Coord., Presses Universitaires de Grenoble, 1978).

Teubner, G., Elementos materiales y reflexivos en el derecho moderno, en La fuerza del derecho, 142 (Nuevo Pensamiento Jurídico, Siglo del Hombre, Pontificia Universidad Javeriana, Uniandes, Bogotá D. C., 2005).

Teubner, G., Regímenes globales privados: ¿derecho neoespontáneo y constitución dual de sectores autónomos? en Estado, soberanía y globalización, 67 y ss. (Siglo del Hombre, Uniandes, Pontificia Universidad Javeriana-Instituto Pensar, Bogotá D. C., 2010).

Twining, W., Cartografiar el derecho, en Derecho y globalización, 173-207 (Siglo del Hombre, Uniandes, Instituto Pensar, Bogotá D. C., 2003).

\section{Artículos}

López Medina, D., El sueño weberiano. Claves para una comprensión constitucional de la estructura administrativa del Estado colombiano, 1 Revista de Derecho Público, 1, 2-36 (2006).

Ordorika Sacristán, I., Educación superior y globalización: las universidades públicas frente a una nueva hegemonía, 3 Revista Andamios, 5, 31 (2006). 


\section{Otras Fuentes}

Sarmiento, J. P., La justicia administrativa entre dictaduras, una crítica a la construcción del derecho administrativo en Colombia (Tesis sin publicar, para optar al título de Doctor en Derecho, Uniandes, 2012). 